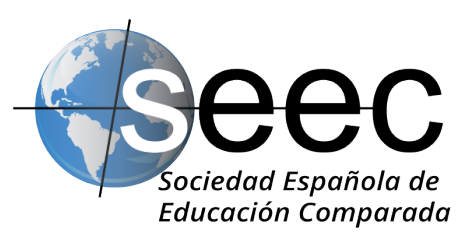

\title{
Uso de herramientas digitales para la docencia en España durante la pandemia por COVID-19
}

\author{
Use of digital tools for teaching in Spain \\ during the COVID-19 pandemic
}

\section{Judit García Martín*; Sheila García Martín**}

DOI: $10.5944 /$ reec.38.2021.27816

\author{
Recibido: 30 de junio de 2020 \\ Aceptado: 14 de octubre de 2020
}

\footnotetext{
* Judit García Martín: Profesora Ayudante Doctora del Departamento de Psicología Evolutiva y de la Educación de la Universidad de Salamanca. Doctora por la Universidad de León cum laude con mención internacional en el Programa de Doctorado en Psicología y Ciencias de la Educación. Datos de contacto: E-mail: jgarm@usal.es. OrciD: https://orcid.org/oooo-00o3-2255-9633

**Sheila García Martín: Profesora Ayudante Doctora en el Área de Didáctica y Organización Escolar. Dpto. de Didáctica General, Específicas y Teoría de la Educación de la Universidad de León. Doctora por la Universidad de León cum laude con mención internacional en el Programa de Doctorado en Psicología Educativa y Ciencias de la Educación. Datos de contacto: E-mail: sgarcm@unileon.es. Orcid: https://orcid. org/oooo-0003-1298-8549
} 


\title{
Resumen
}

Como consecuencia de la pandemia producida por el COVID-19, en marzo de 2020, las instituciones educativas españolas se ven obligadas a suspender las actividades docentes presenciales abocándose a su desempeño de manera virtual, lo que supone un rediseño de las situaciones de enseñanza y de aprendizaje habituales. En este sentido, la digitalización educativa con el uso de herramientas digitales para la docencia, y la competencia digital de los docentes adquieren un papel fundamental en el proceso de enseñanza y de aprendizaje en todos los niveles del sistema educativo español. En este estudio se analiza el uso que hacen 108 docentes en activo, de quince herramientas digitales para la docencia (plataformas educativas, blogs, wikis, herramientas de gamificación, herramientas para crear contenido colaborativo, contenido interactivo, para elaborar encuestas, para grabar audio y video, para editar videos y para programar), durante los meses de confinamiento, y se estudian sus valoraciones respecto al uso de dichas herramientas, a través de la aplicación de un cuestionario online diseñado ad hoc. Al mismo tiempo, se examina la fiabilidad del instrumento y se efectúa un análisis factorial con el que se obtienen nueve componentes que explican el $71.643 \%$ de la varianza total. Seguidamente, se llevan a cabo análisis descriptivos y paramétricos, encontrándose diferencias estadísticamente significativas en el uso de las herramientas digitales cuando se consideran como variables de agrupamiento: el sexo, el funcionariado, la comunidad autónoma y el tipo de centro educativo. A la luz de estos resultados, se discuten y se valoran las implicaciones educativas que han supuesto la pandemia por COVID-19 y el consecuente confinamiento, así como el cambio sustancial producido en el proceso de enseñanza y de aprendizaje con la digitalización educativa materializada en el uso de diversas herramientas digitales para la docencia.

Palabras clave: Enseñanza; Proceso de aprendizaje; Tecnologías de la Información y la Comunicación; Enseñanza a distancia; Telecomunicación.

\begin{abstract}
As a consequence of the pandemic produced by COVID-19, in March 2020, Spanish educational institutions are forced to suspend face-to-face teaching activities by focusing on their performance in a virtual way, which implies a redesign of the usual teaching and learning situations. In this sense, educational digitization with the use of digital tools for teaching, and the digital competence of teachers acquire a fundamental role in the teaching and learning process at all levels of the Spanish educational system. This study analyzes the use made by 108 active teachers of fifteen digital tools for teaching (educational platforms, blogs, wikis, gamification tools, tools to create collaborative content, interactive content, to create surveys, to record audio and video, to edit videos and to program) during the months of confinement, and examines their evaluations regarding the use of these tools, through the application of an online questionnaire designed ad hoc. The reliability of the instrument is examined and a factorial analysis is developed with which nine components are obtained that explain $71.643 \%$ of the total variance. Subsequently, descriptive and parametric analyzes were carried out, finding statistically significant differences in the use of digital tools when were considered as grouping variables: sex, civil service, autonomous community and type of educational center. In light of these results, the educational implications of the COVID-19 pandemic and the consequent confinement, as well as, the substantial change produced in the teaching and learning process with educational digitization materialized in the use of various digital tools for teaching.
\end{abstract}

Keywords: Teaching; Learning process; Information and Communication Technologies; Distance study; Telecommunication. 


\section{Introducción}

Como consecuencia de la situación extrema derivada de la ulterior propagación de la pandemia mundial producida por el coronavirus, COVID-19, en marzo de 2020, las instituciones de enseñanza españolas se ven abocadas a un reto global sin precedentes, la abrupta e inesperada suspensión de las actividades docentes presenciales abocándose a su desempeño de manera virtual, lo que supone un rediseño de las situaciones de enseñanza y de aprendizaje habituales y de los sistemas de evaluación, en todas las etapas educativas. Este rediseño se materializa, en la mayoría de los casos, en la digitalización del contenido mediante el uso de herramientas digitales diversas y en la realización de videoconferencias (sesiones sincrónicas) en los entornos virtuales disponibles, a la misma hora que tendrían lugar las clases presenciales (García-Peñalvo, Corell, Abella-García y Grande-de-Prado, 2020), lo que supone grandes desafíos en un escenario novedoso, en el que proliferan las incertidumbres. Dado que, tal y como exponen Sianes y Sánchez (2020), en cuestión de días, la formación comúnmente conocida se tiene que redirigir al escenario virtual, al mismo tiempo, que se mantiene un equilibrio entre cautela y avance.

Todo ello, ha provocado que, bajo esta emergencia sanitaria global, el profesorado tenga que poner en práctica, metodologías docentes activas innovadoras virtuales de carácter emergente. Lo cual evidencia una vez más, el papel fundamental que desempeña la formación del profesorado del siglo XXI (Ayala y Luzón, 2013), el entrenamiento de los docentes en el uso de herramientas web diversas, así como la promoción de su competencia digital (Gabarda, 2015).

Se trata pues de un hecho histórico mundial que obliga a generar una eficaz correspondencia en términos normativos de la práctica totalidad de las instituciones educativas del territorio geográfico mundial y de las identidades políticas (Sianes y Sánchez, 2020). Además de reflejar, una vez más, las carencias y las desigualdades existentes en torno a la disponibilidad de recursos como a la preparación de los docentes para transitar en la modalidad de educación en línea (Gabarda, 2015; García, 2015; Tiana, 2013). En este sentido, han sido numerosos los organismos internacionales que han realizado informes sobre los retos educativos que esta situación ha planteado.Bajo este contexto social, surgen las siguientes preguntas de investigación: ¿Están los profesores preparados digitalmente para el desempeño de la labor docente de manera virtual?, ¿Qué herramientas digitales utilizan los docentes durante la suspensión de las clases presenciales y durante la instauración del teletrabajo?, ¿Qué valoración realiza el profesorado de las herramientas web examinadas y de su propio uso?

\subsection{La competencia digital docente}

En la actualidad y especialmente durante la última década, ha imperado un constante interés por que los sistemas educativos respondan de manera satisfactoria a las demandas sociales (Tiana, 2013). Por ello, el discente debe ser el centro del método de enseñanza $\mathrm{y}$, a su vez, deben generarse vías de aprendizaje flexibles que garanticen la adquisición de competencias diversas tanto en el alumnado como en el profesorado. Entre dichas competencias se encuentra, la digital (Esteve, Castañeda y Adell, 2018)..

En línea con ello, en España, se delimita, en 2017, un marco común de referencia para el diagnóstico y la optimización de la competencia digital de los docentes a través de la publicación de un minucioso informe elaborado por el Instituto Nacional de Tecnología y Formación del Profesorado (INTEF, 2017) vinculado al Ministerio de Educación, Cultura 
y Deporte (MECD) del Gobierno de España. En él, se hace mención expresa a cinco áreas competenciales en torno a la competencia digital: (i) la información y la alfabetización informacional, (ii) la comunicación y la colaboración, (iii) la creación de contenido digital, (iv) la seguridad, y (v) la resolución de problemas que agrupan veintiuna competencias. Asimismo, se considera que un docente tiene un alto nivel de competencia digital, cuando no solo es capaz de usar las tecnologías para enriquecer sus estrategias didácticas, sino también de proponer y de desarrollar prácticas innovadoras basadas en las posibilidades que le brindan las herramientas digitales (Esteve, Castañeda y Adell, 2018; Gisbert y González, 2016).

En este sentido, la demanda de docentes digitalmente competentes ha ido evolucionando, imponiéndose, en la actualidad, la necesidad de nuevos enfoques cuando se trata de integrar las tecnologías en la educación (Instefjord y Munthe, 2017). De manera que, ser capaz de integrar y utilizar las herramientas digitales en el proceso educativo implica tener un conjunto de habilidades genéricas y específicas de la propia profesión docente (Lund, Furberg, Bakken y Engelien, 2014).Además, durante la última década, se han realizado diversas revisiones sistemáticas y metaanálisis sobre la temática. En este sentido, en un estudio bibliométrico reciente realizado por Rodríguez-García, Raso y RuizPalmero (2019) en el que se analizan, siguiendo los indicadores de calidad establecidos por la declaración PRISMA, ciento cincuenta y cuatro referencias, indexadas en la base de datos de la Web of Science, sobre competencia digital, se evidencia un incremento progresivo de las investigaciones realizadas desde 2014, y se presenta España como el país con mayor producción científica, permitiendo establecer líneas de trabajo sólidas en torno a la adquisición y la promoción de la competencia digital de los agentes de cambio (docentes y discentes) implicados en la situación educativa.

Igualmente, en los últimos años, han acontecido gran cantidad de investigaciones cuantitativas en las que se efectúan, en la mayoría de los casos, estudios experimentales o correlacionales, que han señalado la relación entre el nivel competencial digital de los docentes y el uso que estos hacen de los recursos tecnológicos.

En este sentido, los estudios desarrollados por Law y Chow (2008) y Tejedor y García-Valcárcel (2006) sostienen que los conocimientos que posee el profesorado sobre los recursos tecnológicos constituyen un predictor significativo del uso que realizarán de estos en la escuela. En esta misma línea, Puentes, Roig, Sanhueza y Friz (2010) refieren la existencia de una relación causal entre el conocimiento digital de los docentes y el uso de las tecnologías en el proceso educativo. Así mismo, Condie, Munro, Muir y Collins (2005) informan de que un bajo nivel competencial digital en los docentes se corresponde con un escaso uso de las tecnologías.

Sin embargo, es preciso considerar que la integración de las tecnologías en la educación por parte de los docentes es un proceso complejo en el que intervienen muchos factores, además de su nivel competencial digital. Factores personales de los docentes como la edad, el género, la experiencia profesional, la experiencia en el uso de tecnologías en el proceso de enseñanza y de aprendizaje, así como, factores contextuales relacionados con la institución educativa influyen tanto en la integración de las tecnologías en la educación como en la aceptación de su utilidad por parte de los docentes.

A este respecto, el estudio realizado por Almerich et al. (2016) con maestros de la comunidad autónoma de Valencia evidencia que los factores personales y contextuales tienen un impacto relevante en la adquisición de competencias digitales tales como el uso de las tecnologías de la información y la comunicación (en adelante, TIC) y las actitudes hacia estas. 
Del mismo modo, en el estudio efectuado por Bordalba y Bochaca (2019) en el que se encuestan a 35 docentes acerca de sus creencias de uso del correo electrónico y las plataformas en línea para la comunicación bidireccional entre ellos y los padres, se observa que los docentes tienen actitudes más positivas sobre el uso de estos medios digitales durante el tiempo de permanencia en el centro educativo.

Además, en otro estudio efectuado en 2016 por Carpenter, Tur y Marín, en el que participan 153 docentes en formación a través de la cumplimentación de una encuesta anónima de catorce preguntas cerradas, ocho de las cuales eran escalas tipo Likert de cinco puntos sobre las creencias de uso de Twitter con fines educativos, demuestra que la mayoría de los participantes perciben Twitter como una aplicación educativa útil y manifiestan su intención de utilizarla para fines profesionales en el futuro. Así como, en el estudio realizado por Judit García Martín en 2017, en el que se analizan las percepciones de 483 docentes en formación, a través de la aplicación de siete cuestionarios estandarizados y validados empíricamente en español, se observan patrones de conocimiento, capacitación y uso diferente de las herramientas digitales en función del sexo y del grado de especialización en el que participan.

\subsection{El uso de herramientas digitales para la docencia}

Con relación al uso de las herramientas digitales para el aprendizaje y la docencia, es preciso destacar que los últimos años han sido testigos de la constante incorporación de las tecnologías en el proceso de enseñanza y de aprendizaje.

$\mathrm{Al}$ mismo tiempo, las investigaciones han tratado de conocer las potencialidades educativas de las diversas herramientas, así como, de examinar el uso que los docentes han hecho de las mismas. Algunas tecnologías como los blogs, las wikis, los motores de búsqueda como Google, los archivos de audio y video de YouTube o la mensajería instantánea de WhatsApp han sido objeto de estudio en diversas investigaciones.

Relativo a los blogs, Cano y Cabrera (2013) destacan entre las potencialidades de esta herramienta que permite autorregular el propio proceso de aprendizaje a partir de la reflexión continuada; que ayuda a ampliar las limitaciones clásicas de espacio y de tiempo que se producen en la educación tradicional (Roselló y Pinya, 2017) y que aumenta la satisfacción derivada del aprendizaje, el logro académico y la comprensión del material estudiado (Biberman-Shalev, 2018; Fernández y Pérez, 2015).

Por su parte, las wikis han pasado de ser una herramienta utilizada considerablemente para compartir conocimiento fuera de la escuela, a ser utilizada por algunos docentes, también, en ambientes académicos con el objetivo de apoyar el aprendizaje colaborativo o como herramienta para la enseñanza y el aprendizaje, en general (Gómez, 2017).

Asimismo, las herramientas digitales de Google o de Youtube han sido utilizadas en la docencia de áreas diversas y en etapas educativas diferentes. Herramientas como Google Earth o Google Maps han demostrado ser especialmente útiles en la docencia de asignaturas como Ciencias de la Tierra en la etapa secundaria (Jiménez, Pérez y CarrilloRosúa, 2014), así como los archivos de audio y video de YouTube, en la enseñanza de artes en niveles profesionales (DeWitt, Alias, Siraj, Yaakub, Ayob y Ishak, 2013).

Finalmente, en relación al uso de la mensajería instantánea, a través de aplicaciones como WhatsApp o Voxer, investigaciones previas señalan tanto oportunidades como dificultades. La mensajería instantánea resulta beneficiosa para mejorar las conexiones sociales y favorecer el aprendizaje profesional (Carpenter y Green, 2017), pero la cantidad de tiempo que se dedica al uso de esta herramienta no parece contribuir positivamente a la finalización de las tareas docentes (Junco y Cotten, 2011). 
Por su parte, otras investigaciones de carácter cuantitativo descriptivo-exploratorio tales como el estudio efectuado por Hatlevik (2017) con 312 maestros de escuelas de educación primaria y secundaria, evidencia que el 66,9 \% de los participantes utilizan, durante más de seis horas a la semana, las herramientas digitales con fines administrativos y el 30,4\%, con fines didácticos.

Al mismo tiempo, el estudio descriptivo-interpretativo desarrollado por García Martín (2019b) con 118 docentes de Educación Secundaria Obligatoria, muestra que el $90 \%$ de los profesores encuestados, utilizan herramientas digitales para buscar y seleccionar contenidos educativos, así como ocho de cada diez, utilizan la herramienta digital de Google para planificar y evaluar actividades didácticas.

Sin embargo, a pesar de los diversos estudios desarrollados en relación a la competencia digital de los docentes y al uso de las herramientas digitales en el proceso de enseñanza y de aprendizaje, la situación de excepcionalidad producida por la pandemia COVID-19, el confinamiento y la consecuente instauración del teletrabajo en todos los niveles del sistema educativo español, ha producido una situación, hasta ahora, desconocida, en la que tanto docentes como alumnos se han visto abocados a hacer uso de las herramientas digitales que se encontraban a su alcance, independientemente de su competencia digital, de sus conocimientos y de su valoración sobre la efectividad de las herramientas digitales.

Es en este escenario excepcional, en el que se plantea y se desarrolla el presente estudio que trata de analizar los patrones de uso de diversas herramientas digitales por parte de los docentes durante una situación educativa sin precedentes.

\section{Método}

\subsection{Objetivo e hipótesis}

El objetivo general de este estudio es examinar el uso de quince herramientas digitales por parte del profesorado en activo, durante los meses de confinamiento, así como conocer sus valoraciones respecto a dichas herramientas y al propio uso de las mismas. En base a este objetivo general se plantean las siguientes hipótesis:

H1. El uso y la valoración de las herramientas digitales por parte del profesorado difiere en función de su género.

H2. El uso y la valoración de las herramientas digitales estudiadas se ve condicionado por las variables sociodemográficas: ubicación geográfica, tipo de centro, y, funcionariado.

\subsection{Participantes}

En el mes de abril de 2020, durante la situación de estado de alarma generada a causa de la propagación de la pandemia provocada por el COVID-19, se encuestan, mediante un muestreo no probabilístico incidental y a través de un instrumento online, a un total de 108 docentes españoles en activo, de entre 23 y 65 años. De los cuales, la mayoría desarrolla su labor docente en centros educativos públicos $(69,4 \%)$, no siendo funcionarios de carrera en la mayor parte de los casos $(66,7 \%)$ como puede verse en la tabla 1. 
Tabla 1.

Datos sociodemográficos de la muestra

Hombre $\quad$ Mujer $\quad$ Total

Edad

$\begin{array}{llll}20-35 \text { años } & 15 & 33 & 48 \\ 36-50 \text { años } & 11 & 33 & 44 \\ 51-65 \text { años } & 8 & 8 & 16\end{array}$

Tipo de centro

$\begin{array}{llll}\text { Público } & 21 & 54 & 75 \\ \text { Concertado } & 11 & 13 & 24 \\ \text { Privado } & 2 & 7 & 9 \\ \text { Snario de carrera } & & & \\ \text { Sí } & 13 & 23 & 36 \\ \text { No } & 21 & 51 & 72\end{array}$

Se recogen datos de profesores ejercientes en un total de 13 comunidades autónomas. La mayoría del profesorado encuestado ejerce su labor académica en Castilla y León (39,8 \%), seguido de Cantabria (13 \%), de Cataluña (9,3\%), de Navarra (9,3\%), Madrid (7,4 \%), Andalucía (6,5 \%) y Asturias (6,5\%). Así mismo, en menor medida se recogen datos de docentes de las comunidades de Galicia, Castilla-La Mancha, Extremadura, Comunidad Valenciana, Murcia y La Rioja (ver Figura 1).
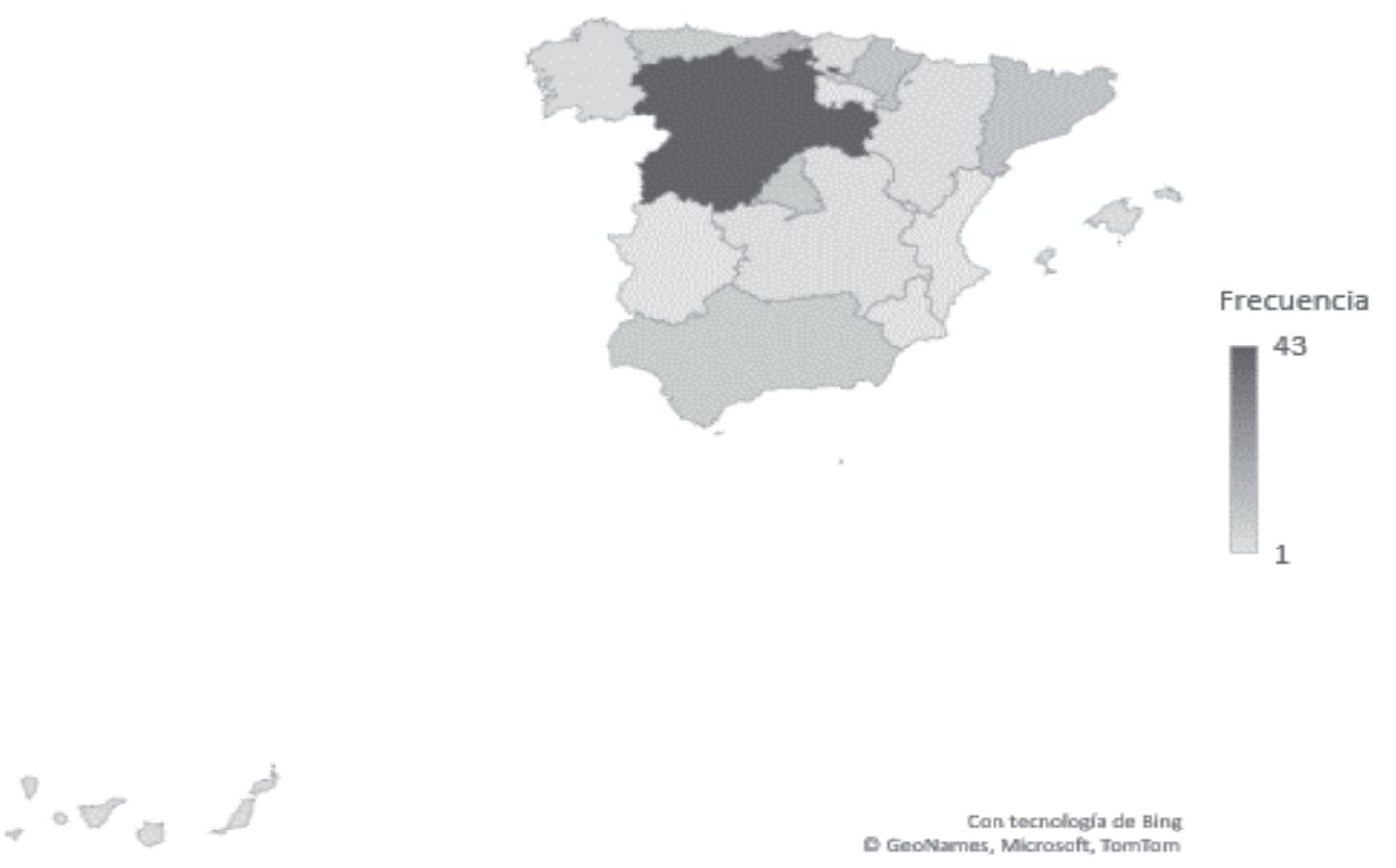

Figura 1: Frecuencia de profesores encuestados por comunidad autónoma 
Asimismo, el 39,4 \% ejercía su labor docente en la Etapa de Educación Primaria, el 32,1 \% en la Universidad, seguido por el profesorado de Educación Secundaria Obligatoria (23,9 \%) y, en menor medida. los de la etapa de Educación Infantil, los de Bachillerato, los de Formación Profesional y los de Formación de Adultos.

\subsection{Diseño}

Este estudio parte de un diseño de investigación de corte cuantitativo, apoyado en un enfoque exploratorio-descriptivo y de correlación, en el que se hace uso del método de encuesta, a través del diseño y de la aplicación de un cuestionario en línea.

\subsection{Instrumento}

Se diseña un cuestionario en línea, denominado: EDU-COVID, a través de la herramienta web de Formularios de Google. Se selecciona dicha herramienta por diversos motivos tales como su gratuidad, su accesibilidad, su simultaneidad, su facilidad, y su eficiencia para la recopilación y la extracción de datos en múltiples formatos. Así como, por la imposibilidad de recoger datos en otro formato, dada la situación excepcional generada por el COVID-19.

El EDU-COVID consta de cinco secciones claramente diferenciadas: i) el consentimiento informado, ii) los datos de carácter general, iii) el uso de quince herramientas web durante el confinamiento, iv) la valoración del uso y, v) los efectos en el proceso de enseñanza-aprendizaje como consecuencia de dicho uso, en un total de 50 ítems.

Para comprobar la fiabilidad del instrumento se procede al cálculo del coeficiente Alfa de Cronbach, medida de consistencia interna, que arroja un valor de $\alpha=.561$ lo que demuestra una fiabilidad aceptable.

Con la intención de valorar la estructura del instrumento en función del conjunto de ítems que lo integran, se realiza un primer Análisis Factorial Exploratorio de sus 43 ítems. La idoneidad del análisis se evalúa previamente a través del test de Kaiser-MeyerOlkin (KMO) y de la prueba de esfericidad de Bartlett.

El resultado de la prueba de adecuación muestral KMO es de .617 , con medidas de KMO individuales de las variables todas superiores a 0.5. Además, la prueba de esfericidad de Bartlett fue estadísticamente significativa ( $\mathrm{p}<$.001), lo que indica que los datos son probablemente factorizables. Por lo tanto, estos resultados revelan que la aplicación del análisis factorial es apropiada.

Seguidamente, se procede a la selección de los principales factores mediante el método de extracción de componentes principales con el objetivo de encontrar una serie de componentes que expliquen el máximo de varianza total de las variables originales. Si se sigue la regla de normalización de Kaiser (1974), que establece la extracción de factores principales de aquellos con valor propio mayor que uno, se obtienen nueve componentes que explican el $71.643 \%$ de la varianza total, lo que puede considerarse un valor muy aceptable.

Para determinar qué ítems saturan en cada uno de los factores, se lleva a cabo la extracción de factores iniciales y se procede a la rotación de factores a través del método de rotación ortogonal Varimax. Las variables, agrupadas por factores, que se obtienen en el análisis y su correspondiente saturación, se presentan en la tabla 2. 
Tabla 2.

Ítems que conforman cada factor y saturación correspondiente

\begin{tabular}{|c|c|c|}
\hline Factor & Ítem & Saturación \\
\hline \multirow{24}{*}{ I } & Uso mecánico / significativo & .645 \\
\hline & Herramientas de edición de videos & .619 \\
\hline & Herramientas de elaboración de encuestas en línea & .617 \\
\hline & Uso superficial / profundo & .614 \\
\hline & Herramientas para videoconferencias & .561 \\
\hline & Uso no funcional / funcional & .555 \\
\hline & Herramientas de grabación de audio y video & .550 \\
\hline & Uso irrelevante / relevante & .538 \\
\hline & Uso insatisfactorio / satisfactorio & .514 \\
\hline & Uso innecesario / necesario & .509 \\
\hline & Herramientas para edición de contenido colaborativo & .503 \\
\hline & Herramientas de almacenamiento en la nube & .487 \\
\hline & Herramientas de creación de contenido interactivo & .485 \\
\hline & Herramientas de gamificación & .471 \\
\hline & Herramientas de programación & .453 \\
\hline & Herramientas de microblogging & .444 \\
\hline & $\begin{array}{l}\text { La enseñanza impartida garantiza la adquisición de las } \\
\text { competencias }\end{array}$ & .431 \\
\hline & $\begin{array}{l}\text { La enseñanza impartida garantiza el logro de los } \\
\text { objetivos }\end{array}$ & .421 \\
\hline & Herramientas para compartir imágenes & .406 \\
\hline & Herramientas de comunicación sincrónica & .401 \\
\hline & Garantiza la obtención de resultados de Aprendizaje & .392 \\
\hline & Uso rígido / flexible & .367 \\
\hline & Google Classroom & .288 \\
\hline & Tipo de centro & .256 \\
\hline \multirow{4}{*}{ II } & Años de experiencia docente & .582 \\
\hline & Edad & .536 \\
\hline & Uso infravalorado / valorado & .389 \\
\hline & Plataforma institucional específica & .380 \\
\hline
\end{tabular}




\begin{tabular}{|c|c|c|}
\hline \multirow{2}{*}{ III } & Redes sociales & .566 \\
\hline & Moodle como plataforma institucional & .341 \\
\hline \multirow{2}{*}{ IV } & Herramientas para el diseño instruccional de wikis & .545 \\
\hline & E-dixgal como plataforma institucional & .536 \\
\hline \multirow{3}{*}{ V } & Provincia & .527 \\
\hline & Classdojo como plataforma institucional & .331 \\
\hline & Comunidad Autónoma & .314 \\
\hline \multirow{3}{*}{ VI } & Sexo & .448 \\
\hline & Herramientas para el diseño instruccional de blogs & .415 \\
\hline & Herramientas de para visualizar y difundir videos & .344 \\
\hline \multirow{3}{*}{ VII } & Escholarium como plataforma institucional & .514 \\
\hline & Funcionario de carrera & .410 \\
\hline & La enseñanza garantiza otro aspecto & .317 \\
\hline VIII & $\begin{array}{l}\text { Formación recibida sobre el uso de herramientas } \\
\text { digitales }\end{array}$ & .402 \\
\hline IX & $\begin{array}{l}\text { La enseñanza impartida garantiza la asimilación de los } \\
\text { contenidos }\end{array}$ & .496 \\
\hline
\end{tabular}

\subsection{Procedimiento}

Se realiza una búsqueda de instrumentos internacionales previos en torno a los bloques examinados con el fin de dar coherencia y articular las variables analizadas en el cuestionario ad hoc. Tras ello, se diseña la herramienta en línea, a través de formularios de Google, y se envía a docentes en ejercicio siguiendo el método Delphi, técnica versátil de recopilación de información basada en la consulta a expertos del área, con el fin de comprobar la funcionalidad, operatividad, así como para eliminar las posibles contrariedades y dificultades que podrían derivarse de la interpretación de los ítems. Los expertos son sometidos individualmente al cuestionario que intercalan con la retroalimentación de lo expresado por el grupo y que, tras sucesivos feedbacks, producen un análisis fiable del cuestionario.

Una vez modificados los ítems ambiguos se procede a su envío al profesorado mediante el correo electrónico, las redes sociales personales y académicas y los grupos docentes existentes en Facebook, LinkedIn y WhatsApp. El enlace está operativo durante el mes de abril. Finalizado el plazo, se procede a la descarga de la matriz resultante, se realizan las codificaciones pertinentes y se llevan a cabo los análisis estadísticos oportunos, mediante el programa SPPS, versión 25, que han aportado las evidencias empíricas de este estudio.

En línea con lo anterior, primeramente, se efectúan unos análisis descriptivos relativos a la media y a la desviación típica para describir a los participantes. Después, se procede con el análisis paramétrico a través de las pruebas de asimetría y curtosis que determinan que la distribución cumple con el supuesto de normalidad. Después, se hacen 
los cálculos de fiabilidad del instrumento, incluyendo la consistencia interna ítem-escala y Alfa de Cronbach. Y, para terminar, se realizan análisis paramétricos a través de las pruebas t Student y ANOVA.

\section{Resultados}

\subsection{Análisis descriptivo}

Se realizan análisis estadísticos descriptivos para conocer el uso de quince herramientas digitales por parte de los docentes y la valoración que estos hacen de las herramientas y del propio uso de las mismas.

En relación al uso de plataformas de enseñanza virtual, se recogen datos acerca de Moodle, Google Classroom, Classdojo, E-dixgal, Escholarium y, otras. De las herramientas mencionadas, son las dos primeras las más utilizadas por los docentes. Tal y como puede observarse en la figura 2, cuatro de cada diez profesores las han utilizado a diario o al menos, semanalmente, durante el estado de alarma.

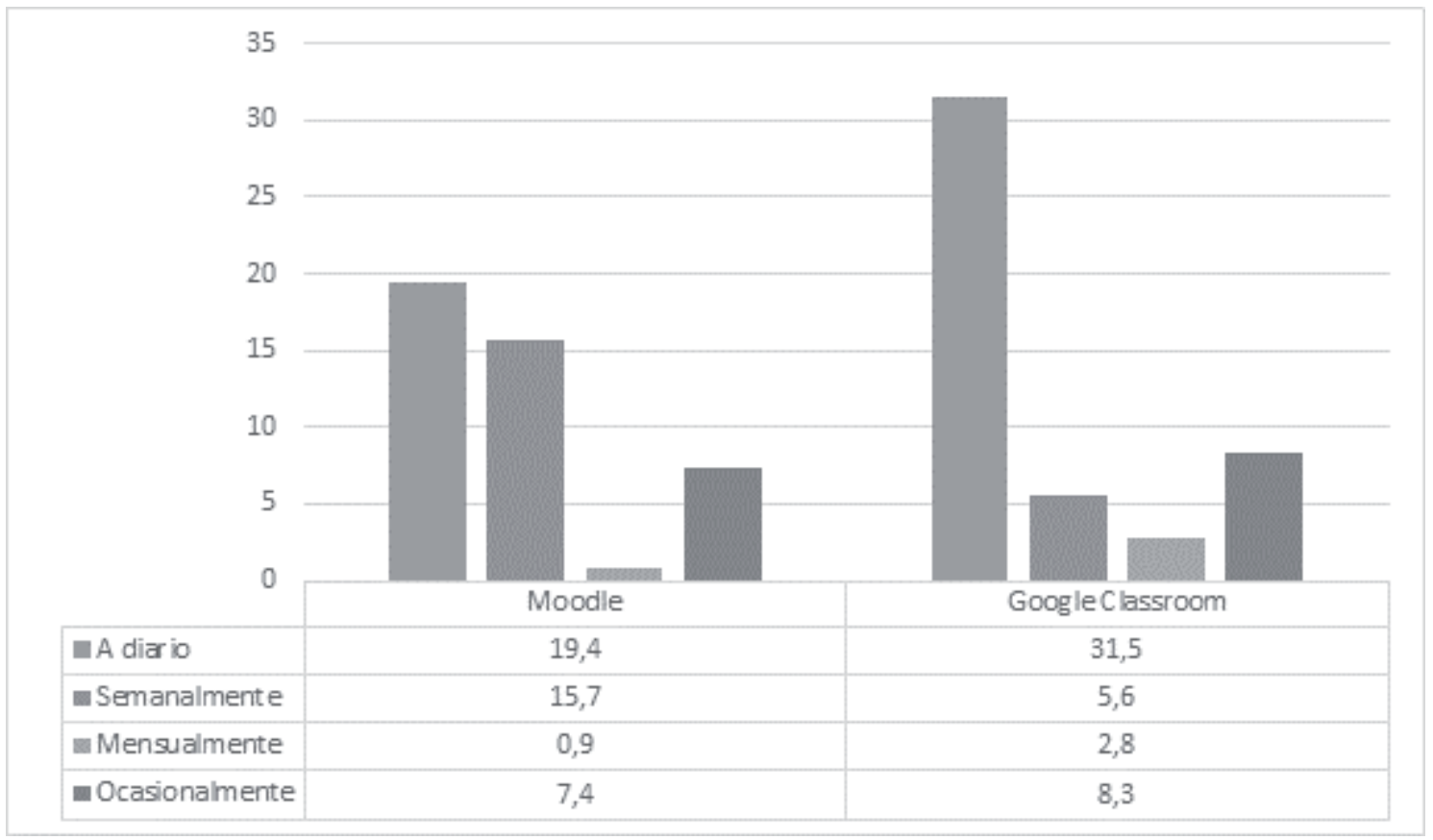

Figura 2: Porcentaje de uso de plataformas de enseñanza virtual

Por su parte, las plataformas Escholarium y E-dixgal no han sido utilizadas en ninguna ocasión por los docentes, en el 98,1 \% y en el 97,2 \% de los casos, respectivamente. Mientras, Classdojo ha sido utilizada diariamente por uno de cada diez profesores. Además, los docentes encuestados afirman utilizar la plataforma específica del centro educativo (5,5 \%), el propio campus virtual (4,6 \%) y Edmodo (3\%). Al mismo tiempo, las herramientas de almacenamiento en la nube, tales como Drive, Dropbox o OneDrive, es utilizada a diario por el $75 \%$ de los docentes encuestados.

Relativo al uso de herramientas digitales o aplicaciones para el diseño instruccional, tal y como puede observarse en la figura 3, son las herramientas de edición de contenido colaborativo como documentos, hojas de cálculo, presentaciones de Google o Prezi, las más utilizadas por los docentes durante el confinamiento. Cerca del $90 \%$ de 
los profesores la han utilizado en alguna ocasión. Seguido del uso de herramientas de elaboración de encuestas en línea a través de Google Formularios o SurveyMonkey, de herramientas de grabación de audio y video como Camstudio o Camtasia, y de creación de contenido interactivo como Canva, Genially, Quizlet o Slideshare.

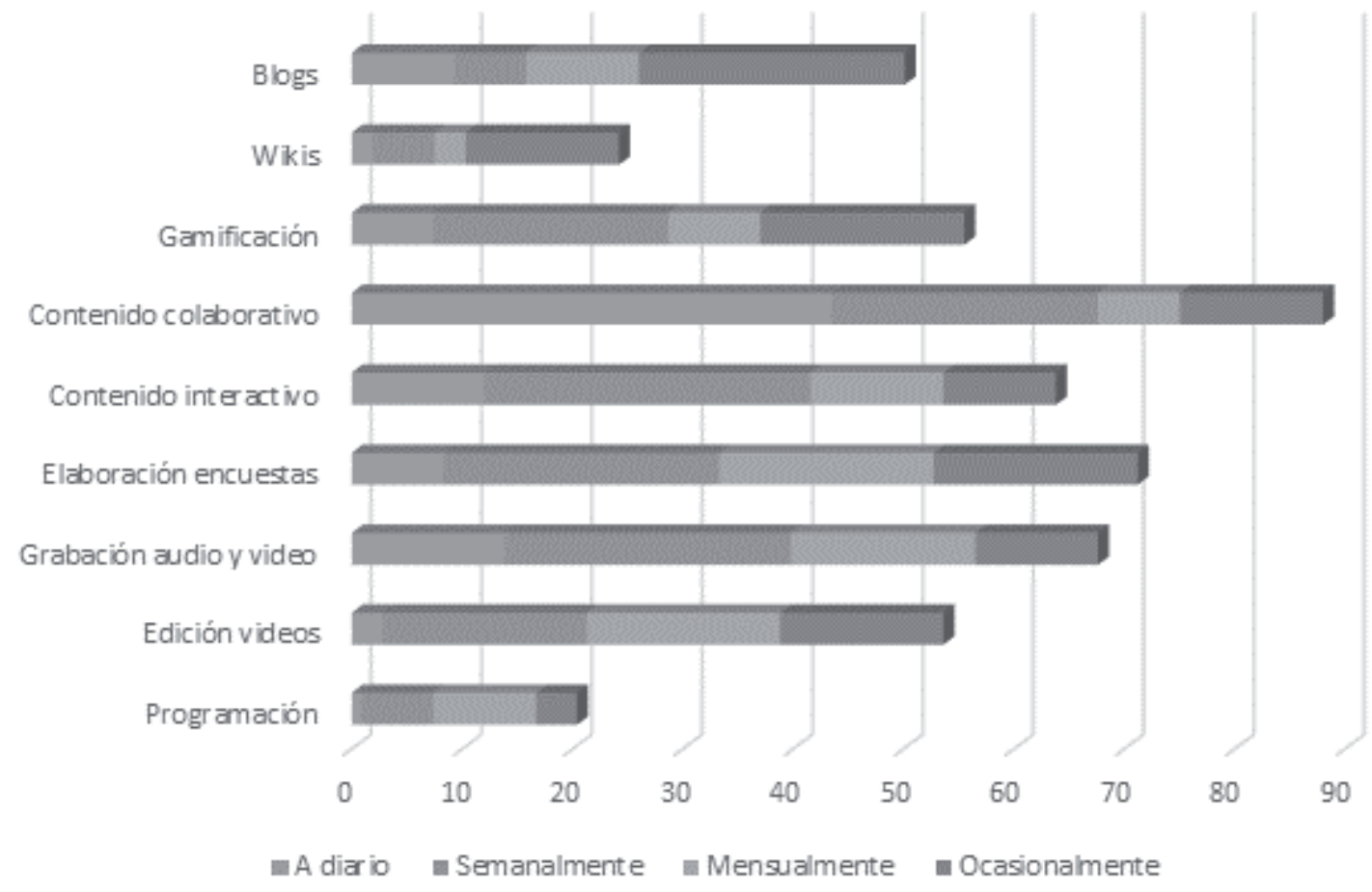

Figura 3: Porcentaje de uso de herramientas digitales o aplicaciones para el diseño instruccional

Finalmente, en relación a la valoración del uso de las herramientas por parte de los docentes, tal y como se observa en la figura 4, el profesorado considera que ha realizado un uso relevante $(96,3 \%)$, necesario $(91,7 \%)$, funcional $(85,2 \%)$ y satisfactorio $(79,6 \%)$ de las herramientas digitales. En sentido opuesto, únicamente seis de cada diez docentes consideran haber realizado un uso profundo de cada una de las herramientas. Así mismo, menos del $60 \%$ cree que dicho uso sea valorado suficientemente.

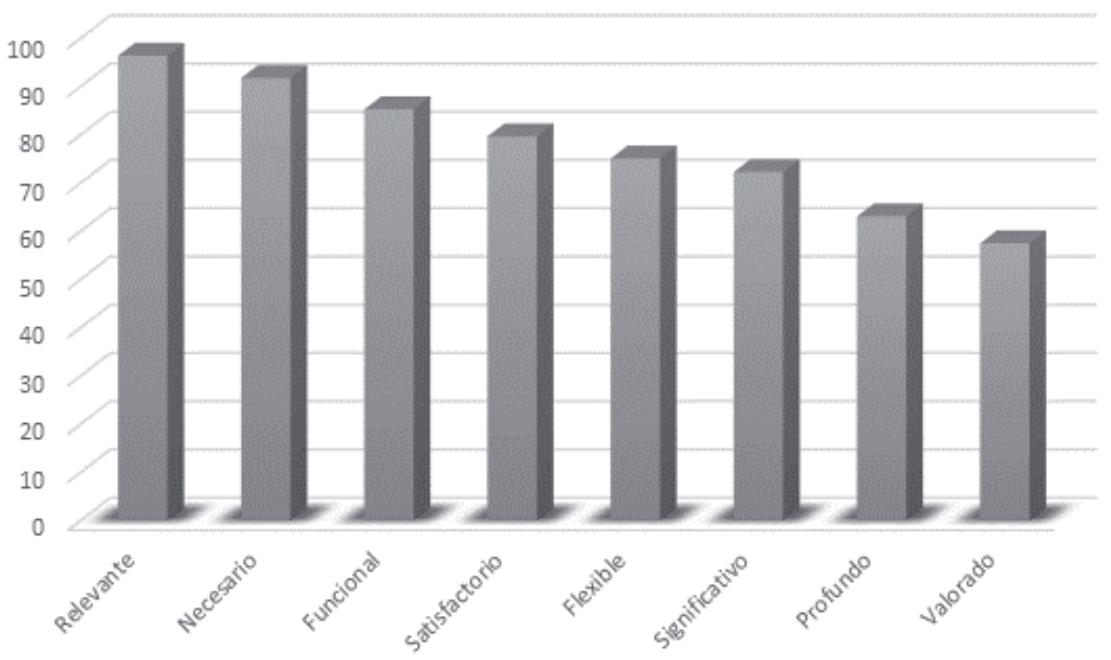

Figura 4: Porcentaje de valoración de uso de herramientas digitales. 


\subsection{Análisis paramétrico}

Se realiza también, el análisis paramétrico que implica una estimación de los parámetros de la población con base en muestras estadísticas, reduciendo así la posibilidad de errores y aumentando el grado de eficiencia. En línea con ello, se efectúan dos tipos de pruebas: i) la t student para muestras independientes y ii) la ANOVA para más de dos muestras independientes.

\subsubsection{Prueba t Student para muestras independientes}

A través de esta prueba, se analizan las variables sexo y funcionario de carrera, y se obtienen diferencias estadísticamente significativas entre poblaciones en relación al uso de las herramientas digitales y a la valoración de las mismas.

Tal y como puede observarse en la tabla 3, existe un patrón de uso diferencial entre los maestros y las maestras en relación a las plataformas de enseñanza virtuales: Moodle, E-dixgal y Escholarium. Es significativamente mayor, en el caso de los docentes varones [ej. MMoodleHombre= 2.76 frente a MMoodleMujer= 2.15; $p=.009$ ]. No observándose dicha tendencia en el caso de Classdojo. Al mismo tiempo, los hombres utilizan en mayor medida las herramientas digitales para la creación de contenido colaborativo, mientras que las mujeres usan más herramientas de gamificación [ej. MGamificMujer= 2.36 frente a $\left.\mathrm{M}_{\text {GamificHombre }}=2.12 ; p=.026\right]$.

Finalmente, en relación a la valoración de las herramientas y del propio uso durante el confinamiento, son las mujeres docentes quienes se sienten más satisfechas. Sin embargo, son los hombres quienes afirman haber realizado un uso más profundo. Los docentes varones consideran que la enseñanza desarrollada durante el estado de alarma ha garantizado la consecución de objetivos y la obtención de resultados de aprendizaje, en mayor medida, que las mujeres.

(Ver Tabla 3 en la siguiente página) 
Tabla 3.

Comparación de las variables examinadas atendiendo al sexo

\begin{tabular}{lccc}
$\begin{array}{c}\text { Variables sobre las creencias } \\
\text { de uso y utilidad }\end{array}$ & Hombre & Mujer & Significación \\
Uso de como plataforma prioritaria de enseñanza virtual & \\
\hline \multirow{2}{*}{ Moodle } & 2.76 & 2.15 & .009 \\
& $(1.843)$ & $(1.594)$ & \\
Classdojo & 1.35 & 1.68 & .027 \\
& $(1.012)$ & $(1.346)$ & .002 \\
E-dixgal & 1.09 & 1.01 & \\
& $(.379)$ & $(.116)$ & .000 \\
Escholarium & 1.06 & 1.00 & \\
& $(.239)$ & $(.000)$ &
\end{tabular}

Uso de herramientas digitales o aplicaciones para el diseño instruccional de...

gamificación tales como Educa-

play, Quizizz, Kahoot, Socrative,

Smile and Learn, Poll Every-

where, Polldaddy...)

$2.12 \quad 2.36$

(1.250) $\quad(1,477)$

.026

contenido colaborativo (Docu-

mentos, Hojas de cálculo y pre-

sentaciones de Google y de Micro-

4.12

soft 360, Prezi)

(1.149)

(1.527)

.000

El uso que has hecho de estas herramientas ha sido...

\begin{tabular}{lccc}
\hline insatisfactorio versus satisfactorio & 4.03 & 4.18 & .016 \\
& $(.717)$ & $(.866)$ & \\
superficial versus profundo & 3.85 & 3.72 & .012 \\
\hline
\end{tabular}

La enseñanza efectuada garantiza la...

\begin{tabular}{|c|c|c|c|}
\hline consecución de objetivos & $\begin{array}{c}1.47 \\
(.507)\end{array}$ & $\begin{array}{c}1.24 \\
(.432)\end{array}$ & .001 \\
\hline $\begin{array}{l}\text { obtención de los resultados de } \\
\text { aprendizaje }\end{array}$ & $\begin{array}{c}1.47 \\
(.507)\end{array}$ & $\begin{array}{c}1.31 \\
(.466)\end{array}$ & .024 \\
\hline
\end{tabular}

Nota. Solo se muestran las variables que obtienen resultados estadísticamente significativos $(p<.05)$.

Por otra parte, cuando se examina la variable funcionario, se obtienen diferencias estadísticamente significativas a favor de los docentes funcionarios en dos variables: el uso de Google Classroom como plataforma prioritaria de enseñanza virtual ( $p=.016$ ) y el uso de herramientas digitales para la elaboración de encuestas en línea tales como los formularios de Google o Mentimeter $(p=.010)$. 


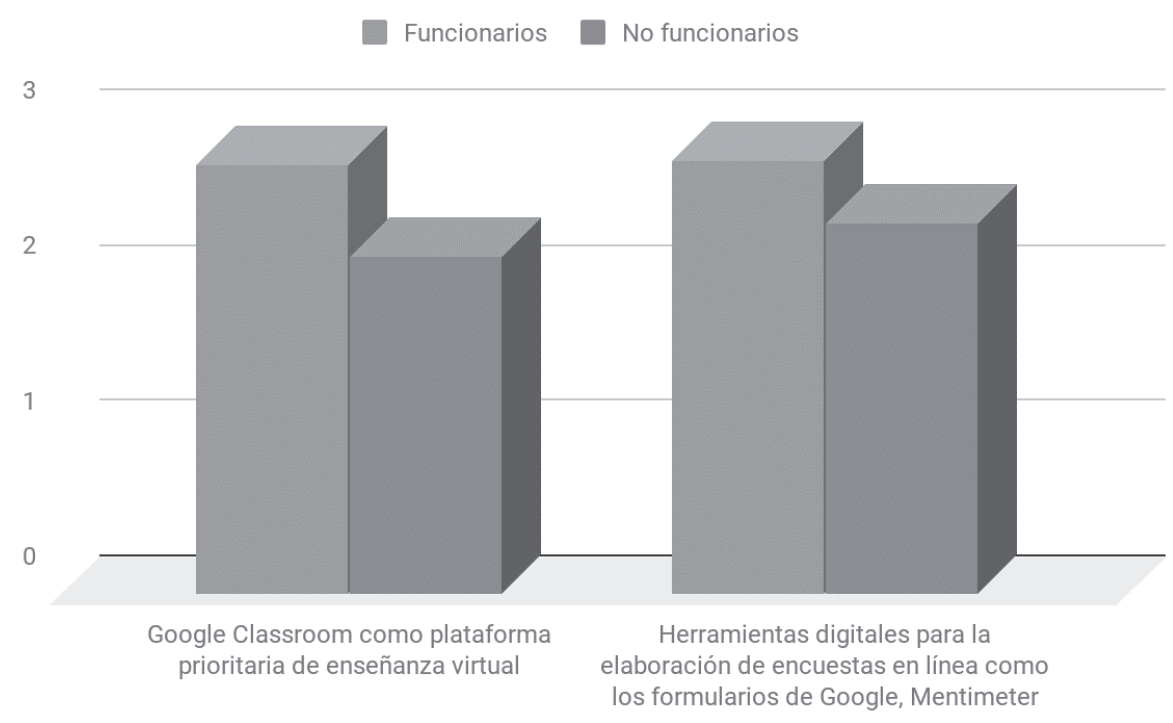

Figura 5: Comparativa de las variables examinada en relación a ser o no funcionario

\subsubsection{Prueba ANOVA para más de dos muestras independientes}

Finalmente, se realizan análisis multivariados de la varianza tomando como factores fijos la comunidad autónoma en la que se ubica el centro educativo, y por tanto en la que ejercen cada uno de los docentes participantes y el tipo de centro (público, concertado y privado). Como variables dependientes, se analizan las medidas relativas a las creencias de uso y de utilidad de las herramientas digitales examinadas a través del cuestionario.

En este sentido, los contrastes multivariados muestran diferencias estadísticamente significativas cuando se atiende a la comunidad autónoma [ $\lambda$ Wilks $=.003, \mathrm{~F}$ $\left.(710.000,420.00)=1.244 ; p=.016, \eta^{2}=.391\right] \mathrm{y}$ al tipo de centro $[\lambda$ Wilks $=.332, \mathrm{~F}$ (142.000, 70.00) $\left.=1.475 ; p=.023, \eta^{2}=.424\right]$ con tamaños del efecto medianos.

En cuanto a los efectos-intersujetos, por una parte, considerando la comunidad autónoma como variable de agrupamiento, se muestran diferencias estadísticamente significativas con tamaños del efecto pequeños, en dos variables: uso superficial versus profundo $\left[p=.043, \eta^{2}=.194\right]$ y uso no funcional versus funcional $\left[p=.032, \eta^{2}=.202\right]$. $Y$, por otra parte, atendiendo al tipo de centro se observan diferencias estadísticamente significativas, con tamaños del efecto medianos y grandes, en las variables relativas al uso de Google Classroom [ $\left.p=.019, \eta^{2}=.073\right]$, de Escholarium $\left[p=.028, \eta^{2}=.066\right]$; de herramientas digitales o aplicaciones para el diseño de encuestas en línea tales como los Formularios de Google, Mentimeter y SurveyMonkey $\left[p=.010, \eta^{2}=.085\right]$ y de herramientas digitales o aplicaciones para programar como Joomla y Scratch [p = $\left..009, \eta^{2}=.086\right]$.

En relación al post hoc, por un lado, en el caso, de la comunidad autónoma, no es posible calcularlo dado que al menos existe un grupo que tiene menos de dos casos. Y, por otro lado, en relación al tipo de centro se evidencia un patrón de uso diferencial entre los docentes de los centros públicos y concertados, en beneficio del profesorado de la enseñanza concertada, en diversas variables tales como el uso de Escholarium como

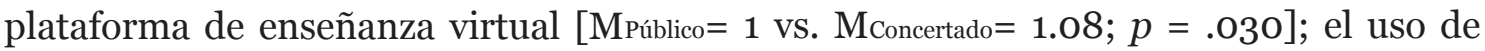
herramientas digitales o aplicaciones para la elaboración de encuestas en línea como los 
Formularios de Google, Mentimeter y SurveyMonkey [MPúblico= 2.48 vs. MConcertado= $3.38 ; p=.016]$ y el uso de herramientas digitales o aplicaciones para programación como Joomla y Scratch $[$ Mrúblico $=1.27$ vs. MConcertado $=1.92 ; p=.015]$.

\section{Conclusiones}

El objetivo del presente estudio fue analizar el uso que los docentes españoles hicieron de quince herramientas digitales durante los meses de confinamiento, y examinar sus valoraciones en relación a dicho uso.

En base al objetivo planteado y a los resultados obtenidos, se observa que las herramientas más utilizadas durante la pandemia fueron las plataformas educativas, Moodle y Google Classroom, lo cual puede deberse a que parte de los docentes encuestados ejercían su labor profesional en niveles de educación superior, en los que Moodle es el sistema de gestión del aprendizaje más utilizado (Del Prete y Cabero, 2020; Martínez-Sarmiento y Gaeta, 2019). Seguido del uso de herramientas de edición de contenido colaborativo entre docentes, de encuestas en línea y de grabación de audio y video. Todo ello puede comprenderse por la necesidad de evaluar o de realizar exámenes en línea, así como, por desarrollar sesiones de docencia magistrales de manera sincrónica o asincrónica a través de la grabación de audio y video (García-Peñalvo, Corell, Abella-García y Grandede-Prado, 2020). Finalmente, las herramientas de creación de contenido interactivo también han sido especialmente utilizadas para la elaboración de material didáctico en todos los niveles de enseñanza.

En relación a la valoración del uso de las herramientas digitales, ocho de cada diez docentes se manifiestan satisfechos con el propio uso, y más del $90 \%$ consideró que este fue relevante, necesario y funcional.

Además, se obtuvieron diferencias estadísticamente significativas en el uso y en la valoración de uso de diferentes herramientas digitales. En relación con el sexo, fueron los docentes varones quienes hicieron un mayor uso de las plataformas educativas y de las herramientas de creación de contenido colaborativo, mientras que las docentes mujeres hicieron un mayor uso de herramientas de gamificación. De manera que, mientras que los varones se focalizan en la practicidad en el uso de herramientas digitales, las mujeres se centran en la motivación e innovación que se presupone al usar otras. Lo que permite afirmar que, aún en situaciones excepcionales, el sexo tiene efectos en el uso diferencial de las tecnologías (García Martín, 2019a; García Martín, 2013; Roig-Vila, Mengual-Andrés y Quinto-Medrano, 2015). Además, dichos resultados también pueden estar relacionados con el modelo de aceptación tecnológica (TAM), en el que la utilidad percibida y la facilidad de uso, son los dos factores más influyentes en la adopción de una tecnología (Fernández, 2016).

Así mismo, las variables socioeducativas tales como funcionariado y tipo de centro educativo influyen en el uso de las herramientas digitales, pues son los docentes funcionarios y los docentes de escuelas concertadas quienes más utilizaron las plataformas educativas durante el confinamiento. Estas diferencias pueden estar condicionadas por las propias políticas educativas en torno al uso e integración de las tecnologías en el proceso de enseñanza y aprendizaje.

Esto corrobora, los hallazgos encontrados en investigaciones como García Martín (2019b); Santiago-Campión, Navaridas-Nalda y Andía-Celaya (2016) y que confirman parcialmente la hipótesis $\mathrm{H} 2$, con relación al funcionariado y a la titularidad del centro, 
no siendo posible confirmar diferencias significativas en el uso y valoración de uso de las herramientas digitales en función de la ubicación geográfica del centro educativo.

De manera general, esta investigación presenta algunas limitaciones. Es un estudio transversal, los datos son recogidos en un único momento temporal y a través de la aplicación de un solo instrumento de recogida de datos. Además, se aplica un muestreo no probabilístico, accediendo únicamente a una parte de la población estudiada. Sería deseable desarrollar investigaciones futuras de carácter longitudinal, con recogida de datos en momentos temporales diversos, muestreos probabilísticos e inclusión de otros datos de análisis tales como el uso didáctico de las herramientas estudiadas y los resultados obtenidos con su aplicación. Así como, datos de procedencia distinta a los autoinformados por los propios docentes.

Finalmente, es preciso concluir que en los últimos años, en los contextos educativos, las mejoras en la enseñanza se han venido relacionando con el uso de las tecnologías en las aulas, de manera que los docentes, en mayor o menor medida, han tenido que desarrollar cierta competencia digital para responder a la demanda de integración de estas herramientas en los procesos de enseñanza y aprendizaje (Almerich, Suárez, Jornet y Orellana, 2011; Law y Chow, 2008; Tejedor y García-Valcárcel, 2006; Vargas-D’Uniam, Chumpitaz-Campos, Suárez-Díaz y Badia, 2014). Sin embargo, la pandemia COVID-19 y el consecuente confinamiento, han producido un cambio sustancial en el proceso de enseñanza y de aprendizaje, dando lugar a una realidad que ha superado cualquier predicción; la tan mencionada digitalización educativa es, hoy en día, una realidad que ha llegado para quedarse.

\section{Referencias}

Almerich, G., Díaz-Costa, I., Cebrián-Cifuentes, S., y Suárez-Rodríguez, J. (2018). Estructura dimensional de las competencias del siglo XXI en alumnado universitario de educación. RELIEVE, 24(1), 1-21. http://doi.org/10.7203/relieve.24.1.12548

Almerich, G., Suárez, J.M., Jornet, J.M., y Orellana, M.N. (2011). Las competencias y el uso de las Tecnologías de Información y Comunicación (TIC) por el profesorado: estructura dimensional. Revista Electrónica de Investigación Educativa, 13(1), 28-42. http://redie.uabc.mx/vol13no1/contenido-almerichsuarez.html

Ayala, A., y Luzón, A. (2013). Presentación: Retos y desafíos de la formación del profesorado en el siglo XXI. Una visión comparada. Revista Española de Educación Comparada, 22, 9-17. https://doi.org/10.5944/reec.22.2013.9320

Biberman-Shalev, L. (2018). Personal blogs or comunal blogs? Pre-service teachers' perceptions regarding the contribution of these two platforms to their professional development. Teaching \& Teacher Education, 69, 253-262. https://doi. org/10.1016/j.tate.2017.10.006

Bordalba, M. M., y Garreta, J. (2019). Digital media for family-school communication? Parents' and teachers' beliefs. Computers \& Education, 132, 44-62. https://doi. org/10.1016/j.compedu.2019.01.006 
Cano, E., y Cabrera, N. (2013). La evaluación formativa de competencias a través de blogs. La experiencia de seis universidades catalanas. Digital Education Review, 23, 46-58. Retrieved from: http://greav.ub.edu/der.

Carpenter, J.P., y Green, T.D. (2017). Mobile instant messaging for professional learning: Educators' perspectives on and uses of Voxer. Teaching \& Teacher Education, 68, 53-97. http://dx.doi.org/10.1016/j.tate.2017.08.008

Carpenter, J., Tur, G., y Marín, V. I. (2016). What do U.S. and Spanish pre-service teachers think about educational and professional use of Twitter? A comparative study. Teaching and Teacher Education, 6o, 131-143. https://doi.org/10.1016/j. tate.2016.08.011

Condie, R., Munro, B., Muir, D., y Collins, R. (2005). The impact of ICT initiatives in Scottish schools: Phase 3. Edimburgo: Scottish Executive Education Departament. Retrieved from: https://www2.gov.scot/Publications/2005/09/14111116/11170

DeWitt, D., Alias, N., Siraj, S., Yaakub, M.Y., Ayob, J., y Ishak, R. (2013). The potential of YouTube for teaching and learning in the performing arts. Procedia. Social and Behavioral Sciences, 103, 118-1126. https://doi.org/10.1016/j.sbspro.2013.10.439

Del Prete, A., y Cabero, J. (2020). El uso del Ambiente Virtual de Aprendizaje entre el profesorado de Educación Superior. RED: Revista de Educación a Distancia, 2O(62). https://doi.org/10.6018/red.400061

Esteve, F., Castañeda, L., y Adell, J. (2018). Un modelo holístico de competencia docente para el mundo digital. Revista interuniversitaria de formación del profesorado, 91(32.1), 105-116.

Fernández, B. (2016). Adopción de la realidad aumentada por estudiantes del grado de Educación Primaria. En Roig-Vila (Edit.). Tecnología, innovación e investigación en los procesos de enseñanza-aprendizaje (pp.432-439). Barcelona: Octaedro.

Fernández, V., y Pérez, S. (2015). Los blogs en la educación. Ejemplo práctico. Tecnología, Ciencia y Educación. Revista de Carácter Científico Multidisciplinar, 2, 30-38. https://dialnet.unirioja.es/descarga/articulo/6159626.pdf

Gabarda, V. (2015). Uso de las TIC en el profesorado europeo, ¿una cuestión de equipamiento y formación? Revista Española de Educación Comparada, 26, 153170. https://doi.org/10.5944/reec.26.2015. 14448

García, R. (2015). Desafíos a la igualdad en el siglo XXI: dificultades y retos del modelo de escuela comprensiva. Revista Española de Educación Comparada, 26, 135-152. https://doi.org/10.5944/reec.26.2015. 14445

García-Martín, J. \& García-Sánchez, J. N. (2013). Patterns of Web 2.0 tool use among young Spanish people. Computers \& Education, 67, 105-120. https://doi. org/10.1016/j.compedu.2013.03.003

García-Martín, J. \& García-Sánchez, J. N. (2017). Pre-service teachers' perceptions of the competence dimensions of digital literacy and of psychological and educational measures. Computers \& Education, 107, 54-67. https://doi.org/10.1016/j. compedu.2016.12.010 
García-Martín, S. \& Cantón-Mayo, I. (2019a). Uso de tecnologías y rendimiento académico en estudiantes adolescentes. Comunicar, 59, 73-81. https://doi.org/10.3916/C592019-07.

García-Martín, S. \& Cantón-Mayo, I. (2019b). Teachers 3.0: Patterns of use of five digital tools. Digital Education Review, 35, 202-215. https://doi.org/10.1344/ der.2019.35.202-215

García-Peñalvo, F. J., Corell, A., Abella-García, V., y Grande-de-Prado, M. (2020). Online Assessment in Higher Education in the time of COVID-19. Education in the Knowledge Society, 21(12), 1-26. https://doi.org/10.14201/eks.23013

Gisbert, M., y González, J. (2016). Nous escenaris d'aprenentatge des d'una visió transformadora. Wolters Kluwer.

Gómez, V. (2017). ¿Cómo se involucran los estudiantes de pedagogía en una experiencia de blogging internacional en wikispaces?. Digital Education Review, 31, 1-19. http://greav.ub.edu/der/

Hatlevik, O. E. (2017). Examining the Relationship between Teachers' Self-Efficacy, their Digital Competence, Strategies to Evaluate Information, and use of ICT at School. Scandinavian Journal of Educational Research, 61(5), 555-567. https://doi.org/1 0.1080/00313831.2016.1172501

Instefjord, E.J., y Munthe, E. (2017). Educating digitally competent teachers: A study of integration of professional digital competence in teacher education. Teaching and teacher education, 67, 37-45. http://dx.doi.org/10.1016/j.tate.2017.05.016

Instituto Nacional de Tecnologías Educativas y de Formación del Profesorado (2017). Marco común de competencia digital docente. INTEF. https://aprende. intef.es/sites/default/ files/2018-05/2017_1020_Marco-Com\%C3\%BAn-deCompetencia-Digital-Docente.pdf

Jiménez, A., Pérez, J.V., y Carrillo-Rosúa, J. (2014). Integración de las Tecnologías Geoespaciales como herramientas docentes de Ciencias de la Tierra para Educación Secundaria. Enseñanza de las Ciencias de la Tierra, 22(3), 239-250. http:// digibug.ugr.es/handle/10481/39130

Junco, R., y Cotten, S.R. (2011). Perceived academic effects of instant messaging use. Computers \& Education, 56, 370-378. http://dx.doi.org/10.1016/j. compedu.2010.08.020

Law, N., y Chow, A. (2008). Teacher characteristics, contextual factors, and how these affect the pedagogical use of ICT. Pedagogy and ICT Use, 181-219. https://doi. org/10.1007/978-1-4020-8928-2_6

Lund, A., Furberg, A., Bakken, J., y Engelien, K. L. (2014). What does professional digital competence mean in teacher education? Nordic Journal of Digital Literacy, 9(4), 281-299.

Martínez-Sarmiento, L. F., y Gaeta, M.L. (2019). Utilización de la plataforma virtual Moodle para el desarrollo del aprendizaje autorregulado en estudiantes universitarios. Educar, 55(2), 479-498. https://doi.org/10.5565/rev/educar.883 
Puentes, A., Roig, R., Sanhueza, S., y Friz, M. (2013). Concepciones sobre las tecnologías de la información y la comunicación (TIC) y sus implicaciones educativas: Un estudio exploratorio con profesorado de la provincia de Ñuble, Chile. Revista CTS, 8(22), 7588. http://www.revistacts.net/files/Volumen\%208\%2020N\%C3\%BAmero\%20 22/SanhuezaDEF.pdf

Rodríguez-García, A-M., Raso, F., y Ruiz-Palmero, J. (2019). Competencia digital, educación superior y formación del profesorado: un estudio de meta-análisis en la Web of Science. PIXEL BIT. Revista de Medios y Educación, 54, 65-81. https:// doi.org/10.12795/pixelbit.2019.i54.04

Roig-Vila, R., Mengual-Andrés, S., y Quinto-Medrano, P. (2015). Conocimientos tecnológicos, pedagógicos y disciplinares del profesorado de Primaria. Comunicar, 45(23), 151-159. http://dx.doi.org/10.3916/C45-2015-16

Rosselló, M., y Pinya, C. (2017). Using blogs to improve profesional competencies among undergraduate students. Digital Education Review, 31, 20-38. http://greav. ub.edu/der/

Santiago-Campión, R., Navaridas-Nalda, F., y Andía-Celaya, L.A. (2016). Las percepciones de los directivos de centros escolares sobre el uso y el valor de las TIC para el cambio e innovación educativa. Estudios sobre Educación, 30, 145-174. https://doi.org/10.15581/004.30.145-174

Sianes, A., y Sánchez, E. (2020). E-learning en 15 días. Retos y renovaciones en la Educación Primaria y Secundaria de la República de Croacia durante la crisis del COVID-19. How have we introduced distance learning? Revista Española de Educación Comparada, 36, 181-195. https://doi.org/10.5944/reec.35.2020.26208

Tejedor, F., y García-Valcárcel, A. (2006). Competencias de los profesores para el uso de las TIC en la enseñanza. Análisis de sus conocimientos y actitudes. Revista Española de Pedagogía, 223, 21-44. https://dialnet.unirioja.es/servlet/ articulo?codigo $=1973261$

Tiana, A. (2013). Los cambios recientes en la formación inicial del profesorado en España: una reforma incompleta. Revista Española de Educación Comparada, 22, 39-58. https://doi.org/10.5944/reec.22.2013.9322

Vargas-D’Uniam, J., Chumpitaz-Campos, L., Suárez-Díaz, G., y Badia, A. (2014). Relación entre las competencias digitales de docentes de educación básica y el uso educativo de las tecnologías en las aulas. Profesorado. Revista de Currículum y Formación del Profesorado, 18(3), 361-377. http://www.ugr.es/local/recfpro/rev183COL9.pdf 


\section{Anexo}

\section{La adaptación de la enseñanza a la situación actual y el uso de las herramientas digitales}

\section{PRESENTACIÓN}

Estimado docente,

La enseñanza online y el teletrabajo se han constituido como la forma de proceder en todos los niveles educativos debido a la situación actual, generada por el COVID-19.

Ante esta situación, investigadores de la Universidad de Salamanca y de la Universidad de León están desarrollando un estudio sobre el uso de las herramientas digitales por parte de los docentes y el efecto de este uso sobre su labor profesional.

Agradeciendo su respuesta.

Reciba saludos cordiales.

\section{CONSENTIMIENTO INFORMADO}

1. Declaro que soy mayor de edad.

2. Confirmo que he leído y comprendo la información anterior y que se me ha informado de la libertad de retirar mi consentimiento y poner fin a mi participación en cualquier momento.

3. Doy mi consentimiento para participar en este estudio con el entendimiento de que dicho consentimiento no conlleva la renuncia a ninguno de mis derechos legales.

4. Afirmo que he leído y he comprendido la información brindada sobre la protección de datos y doy mi consentimiento para que la información sea recolectada y almacenada electrónicamente. Declaro ser consciente también que mediante el envío del cuestionario doy mi consentimiento para el uso de los datos que se almacenarán de forma anónima, y que el acceso y la supresión de los datos facilitados no será posible a partir de este momento.

Declaración de consentimiento Sí / No

\section{DATOS DE CARÁCTER GENERAL}

1. Identificador (Las últimas cuatro cifras de tu número de teléfono móvil)

2. Sexo (Hombre y mujer)

3. Edad (23-70)

4. Funcionario de carrera (Sí - No)

5. Años de experiencia docente (0-50)

6. Etapa educativa en la que imparte docencia (Infantil, Primaria, Secundaria, Bachillerato, FP, Universidad, Formación de Adultos)

7. Tipo de centro (Público, Privado, Concertado)

8. Si se trata de un colegio público, indicar tipo. (CRA, CEIP, CEO, IES, IESO, Centro de Adultos, Universidad)

9. Comunidad Autónoma

10. Provincia en la que se ubica el centro 


\section{USOS}

\section{Durante el confinamiento, en qué medida has utilizado...}

\section{1. ...la plataforma institucional de enseñanza virtual?}

$\begin{array}{llllll}\begin{array}{l}\text { Moodle } \\ \text { Google } \\ \text { Classroom }\end{array} & \text { Nunca } & \text { Ocasionalmente } & \text { Mensualmente } & \text { Semanalmente } & \text { A diario } \\ \text { Classdojo } & \text { Nunca } & \text { Ocasionalmente } & \text { Mensualmente } & \text { Semanalmente } & \text { A diario } \\ \text { E-dixgal } & \text { Nunca } & \text { Ocasionalmente } & \text { Mensualmente } & \text { Semanalmente } & \text { A diario } \\ \text { Escholarium } & \text { Nunca } & \text { Ocasionalmente } & \text { Mensualmente } & \text { Semanalmente } & \text { A diario } \\ \text { Otra } & \text { Nunca } & \text { Ocasionalmente } & \text { Mensualmente } & \text { Semanalmente } & \text { A diario } \\ & \text { Nunca } & \text { Ocasionalmente } & \text { Mensualmente } & \text { Semanalmente } & \text { A diario }\end{array}$

2. ...las herramientas de almacenamiento en la nube (Drive, OneDrive, Dropbox...)?

\section{Nunca / Ocasionalmente / Mensualmente / Semanalmente / A diario}

3. ...las siguientes herramientas digitales o aplicaciones para el diseño instruccional de:

\begin{tabular}{|c|c|c|c|c|c|}
\hline $\begin{array}{l}\text { blogs (Blogger, } \\
\text { Wordpress...) }\end{array}$ & Nunca & $\begin{array}{l}\text { Ocasional- } \\
\text { mente }\end{array}$ & $\begin{array}{l}\text { Mensual- } \\
\text { mente }\end{array}$ & Semanalmente & A diario \\
\hline $\begin{array}{l}\text { wikis (Wikispaces, } \\
\text { TiddlyWiki, Mediawiki...) }\end{array}$ & Nunca & $\begin{array}{l}\text { Ocasional- } \\
\text { mente }\end{array}$ & $\begin{array}{l}\text { Mensual- } \\
\text { mente }\end{array}$ & Semanalmente & A diario \\
\hline $\begin{array}{l}\text { gamificación (Educaplay, } \\
\text { Quizizz, Kahoot, Socrative, } \\
\text { Smile and Learn, Poll } \\
\text { Everywhere, Polldaddy...) }\end{array}$ & Nunca & $\begin{array}{l}\text { Ocasional- } \\
\text { mente }\end{array}$ & $\begin{array}{l}\text { Mensual- } \\
\text { mente }\end{array}$ & Semanalmente & A diario \\
\hline $\begin{array}{l}\text { edición de contenido } \\
\text { colaborativo (Documentos, } \\
\text { Hojas de cálculo y } \\
\text { presentaciones de Google y } \\
\text { de Microsoft 360, Prezi) }\end{array}$ & Nunca & $\begin{array}{l}\text { Ocasional- } \\
\text { mente }\end{array}$ & $\begin{array}{l}\text { Mensual- } \\
\text { mente }\end{array}$ & Semanalmente & A diario \\
\hline $\begin{array}{l}\text { creación de contenido } \\
\text { interactivo (Canva, Genially, } \\
\text { Liveworksheets, Quizlet, } \\
\text { Prezi, Slideshare...). }\end{array}$ & Nunca & $\begin{array}{l}\text { Ocasional- } \\
\text { mente }\end{array}$ & $\begin{array}{l}\text { Mensual- } \\
\text { mente }\end{array}$ & Semanalmente & A diario \\
\hline $\begin{array}{l}\text { elaboración de encuestas } \\
\text { en línea (Formularios } \\
\text { de Google, Mentimeter, } \\
\text { SurveyMonkey...) }\end{array}$ & Nunca & $\begin{array}{l}\text { Ocasional- } \\
\text { mente }\end{array}$ & $\begin{array}{l}\text { Mensual- } \\
\text { mente }\end{array}$ & Semanalmente & A diario \\
\hline $\begin{array}{l}\text { grabación de audio y video } \\
\text { (Camstudio, Screencast-o- } \\
\text { matic, Camtasia...) }\end{array}$ & Nunca & $\begin{array}{l}\text { Ocasional- } \\
\text { mente }\end{array}$ & $\begin{array}{l}\text { Mensual- } \\
\text { mente }\end{array}$ & Semanalmente & A diario \\
\hline $\begin{array}{l}\text { edición de vídeos } \\
\text { (IMovie,FinalCut) }\end{array}$ & Nunca & $\begin{array}{l}\text { Ocasional- } \\
\text { mente }\end{array}$ & $\begin{array}{l}\text { Mensual- } \\
\text { mente }\end{array}$ & Semanalmente & A diario \\
\hline $\begin{array}{l}\text { programación (Jommla, } \\
\text { Scratch) }\end{array}$ & Nunca & $\begin{array}{l}\text { Ocasional- } \\
\text { mente }\end{array}$ & $\begin{array}{l}\text { Mensual- } \\
\text { mente }\end{array}$ & Semanalmente & A diario \\
\hline
\end{tabular}


4. ...las siguientes herramientas digitales de comunicación y de interacción social para:

comunicación sincrónica

(WhatsApp, Telegram, Bloomz)

$\begin{array}{lllll}\text { Nunca } & \text { Ocasionalmente } & \text { Mensualmente } & \text { Semanalmente A diario } \\ \text { Nunca } & \text { Ocasionalmente } & \text { Mensualmente } & \text { Semanalmente A diario } \\ \text { Nunca } & \text { Ocasionalmente } & \text { Mensualmente } & \text { Semanalmente A diario } \\ \text { Nunca } & \text { Ocasionalmente } & \text { Mensualmente } & \text { Semanalmente A diario } \\ \text { Nunca } & \text { Ocasionalmente } & \text { Mensualmente } & \text { Semanalmente A diario } \\ \text { Nunca } & \text { Ocasionalmente } & \text { Mensualmente } & \text { Semanalmente A diario }\end{array}$

videoconferencias

(FaceTime, Skype, Google

Meet, Microsoft Teams,

Zoom, Webinar, Cisco

Webex, Adobe Connect,

Hangouts...)

visualizar y difundir videos

(YouTube, Vimeo...)

compartir imágenes

(Instagram, Flickr,

Picassa...)

relacionarse a través de redes sociales (Facebook, LinkedIn...)

microblogging (Twitter, Tumblr)

5. ¿En qué medida considera que el uso realizado de estas herramientas, durante el confinamiento, ha sido...?

\begin{tabular}{|c|c|c|c|c|c|c|}
\hline Irrelevante & 1 & 2 & 3 & 4 & 5 & Relevante \\
\hline Insatisfactorio & 1 & 2 & 3 & 4 & 5 & Satisfactorio \\
\hline Superficial & 1 & 2 & 3 & 4 & 5 & Profundo \\
\hline Mecánico & 1 & 2 & 3 & 4 & 5 & Significativo \\
\hline Innecesario & 1 & 2 & 3 & 4 & 5 & Necesario \\
\hline No funcional & 1 & 2 & 3 & 4 & 5 & Funcional \\
\hline Rígido & 1 & 2 & 3 & 4 & 5 & Flexible \\
\hline Infravalorado & 1 & 2 & 3 & 4 & 5 & Valorado \\
\hline
\end{tabular}

6. Considera que la enseñanza online, impartida durante el confinamiento, garantiza...

- la adquisición de las competencias.

- el logro de los objetivos.

- la asimilación de contenidos.

- la obtención de resultados de aprendizaje.

- otra.

7. ¿Ha recibido formación online sobre el uso de algunas herramientas digitales mencionadas anteriormente? Sí / No

En caso afirmativo, indique cuál...

8. Si en el futuro, tuviese la oportunidad de recibir formación online sobre herramientas digitales, ¿̇cuál de las mencionadas con anterioridad sería su preferida? 